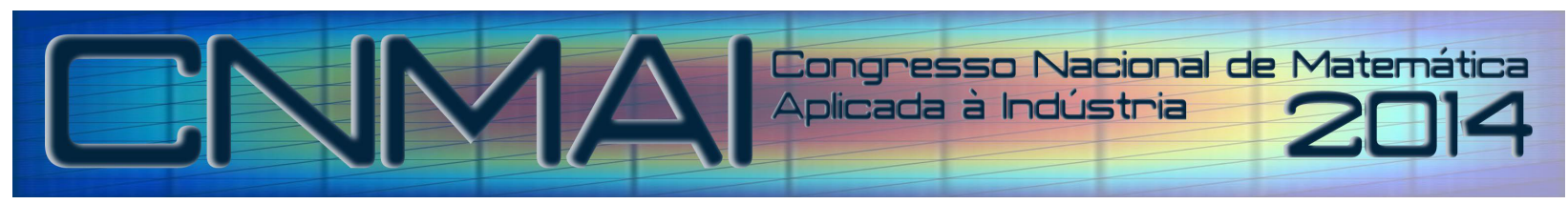

18 a 21 de novembro de 2014, Caldas Novas - Goiás

\title{
INVESTIGAÇÃO NUMÉRICA-EXPERIMENTAL DA INFLUÊNCIA DE PRÉ-CARGAS ESTÁTICAS COMBINADA À CARREGAMENTOS CÍCLICOS SOBRE O FENÔMENO DO AUTOAQUECIMENTO EM MATERIAIS VISCOELÁSTICOS
}

\author{
Luiz Fernando Ferreira Rodovalho, luiz.ffrodovalho@gmail.com ${ }^{1}$ \\ Antonio Marcos Gonçalves de Lima, amglima@ mecanica.ufu.br ${ }^{1}$ \\ Romes Antonio Borges, romes.borges@ catalao.ufg.br² \\ Núbia dos Santos Saad, nubia@ufu.br ${ }^{1}$ \\ Helder Barbieri Lacerda, helder@mecanica.ufu.br ${ }^{1}$
}

\begin{abstract}
${ }^{1}$ Universidade Federal de Uberlândia - Faculdade de Engenahria Mecânica, Campus Santa Mônica, Av. João Naves de Ávila, 2121, C.P. 593, CEP. 38400-902, Uberlândia-MG, Brasil.

${ }^{2}$ Universidade Federal de Goiás - Departamento de Matemática e Matemática Industrial, Regional Catalão, Av. Dr. Lamartine Pinto de Avelar, 1120, CEP. 75704-020, Catalão-GO, Brasil.
\end{abstract}

\begin{abstract}
Resumo: Devido às inúmeras possibilidades de aplicações em engenharia, a busca pelo amplo domínio tecnológico sobre o mecanismo interno de amortecimento dos materiais viscoelásticos, recorre ao desenvolvimento de metodologias de modelagem e ferramentas numéricas de simulação, cada vez mais efetivas e inclusivas da totalidade dos efeitos induzidos por parâmetros operacionais e ambientais. Neste sentido, seja na concepção inicial ou na fase de pré-projeto de sistemas, integrando componentes viscoelásticos, o fenômeno do autoaquecimento, muitas vezes negligenciado na maioria dos procedimentos tradicionais de análise, deve ser levado em conta na caracterização $e$ predição do comportamento estrutural, pois, pode representar expressivas perdas de desempenho e significativas alterações das respostas dinâmicas da estrutura em questão. Para aplicações envolvendo a submissão simultânea do material viscoelástico a certo pré-carregamento estático combinado à cargas dinâmicas cíclicas, como coxins de motores e configurações de isolamento ou amortecimento relacionados à altas frequências, tais procedimentos podem levar à projetos pobres ou mesmo, à graves falhas, uma vez que a dissipação de energia pelo material conduz a elevação da temperatura interna do mesmo. Neste trabalho, efetiva-se uma investigação numérica-experimental dos efeitos de pré-cargas estáticas sobre o fenômeno do autoaquecimento em amortecedores viscoelásticos carregados ciclicamente, objetivando especificamente o fenômeno da fuga térmica (thermal runaway) para um completo estado triaxial de tensões. Posteriormente à apresentação dos principais aspectos teóricos da metodologia, os resultados numéricos e experimentais em termos da evolução da temperatura serão comparados para diferentes níveis de précargas estáticas.
\end{abstract}

Palavras-chave: Materiais viscoelásticos, autoaqeucimento, termoviscoelasticidade, fuga térmica

\section{INTRODUÇÃ̃O}

No contexto das técnicas de controle passivo de ruídos e vibrações de sistemas mecânicos, a capacidade dissipativa dos materiais viscoelásticos vêm sendo paulatinamente explorada por muitos autores no aumento do amortecimento estrutural. Com vistas às inúmeras possibilidades de aplicações práticas em engenharia, a busca pelo controle desta tecnologia tem levado ao desenvolvimento de técnicas mais eficientes para a modelagem numérica e caracterização experimental do comportamento elastodinâmico de sistemas incorporando tais materiais, uma vez que, a maioria dos procedimentos tradicionais de análise e projeto, assumem uma distribuição de temperatura uniforme e constante no volume do material, negligenciando o fenômeno do autoaquecimento, cuja importância relativa pode resultar em aumentos significativos e não homogêneos da temperatura interna do material viscoelástico afetando significativamente a capacidade dissipativa e o comportamento dinâmico estrutural. 
A conveniência de uma metodologia baseada em elementos finitos, com capacidade de tratamento de geometrias complexas e completos estados triaxiais de tensão-deformação é de grande interesse na avaliação da distribuição de temperatura e preditividade dos efeitos do fenômeno do autoaquecimento. Avanços já obtidos neste cenário, consistem de procedimentos de modelagem numérico-computacionais e caracterização experimental da influência simultânea da frequência de excitação e dos efeitos da temperatura sobre composições viscoelásticas (Rittel, 1999; Cazenove et al, 2012). No entanto, em aplicações combinando pré-cargas estáticas à esforços dinâmicos cíclicos, tais como coxins de motores e configurações de isolamento ou amortecimento à altas frequências, a determinação da resposta estática e dinâmica combinadas em termos da distribuição e da evolução do campo de temperatura em materiais viscoelásticos enseja uma problema de grande relevância cientifico-tecnológica acerca da potencialidade desta sobreposição no autoaquecimento e na dinâmica íntegro-estrutural (Nashif et al, 1985).

Neste trabalho é realizada a avaliação numérico-computacional dos efeitos induzidos por pré-cargas estáticas sobre o fenômeno do autoaquecimento interno em materiais viscoelásticos, enfocando-se o fenômeno da fuga térmica para um completo estado tridimensional de tensões. O procedimento operacional para verificação experimental do mesmo é descrito e os resultados em termos da evolução da temperatura são apresentados e comparados aos valores obtidos com as simulações numéricas, detalhando-se a metodologia de identificação paramétrica via utilização da técnica Colônia de Vagalumes (Yang, 2008) para o ajuste do modelo numérico.

\section{REPRESENTAÇÃO DA FUNÇÃo MÓDULO COMPLEXO PARA OS MÓDULOS ESTÁTICO E DINÂMICO COMBINADOS}

As propriedades mecânicas dos materiais viscoelásticos dependem de inúmeros fatores, dentre os quais a frequência de excitação e a temperatura tem sido mais relevantemente considerados na modelagem matemática da viscoelasticidade linear (Nashif et al, 1985). Diversos modelos já foram propostos para a representação do comportamento viscoelásticos sendo considerados bem adaptados para a utilização conjunta com o método dos elementos finitos. Entretanto, em aplicações em que o material viscoelástico é submetido simultaneamente a esforços mecânicos cíclicos sobrepostos à pré-cargas estáticas, é necessário que o modelo seja suficientemente representativo dos efeitos combinados das deformações estáticas e dinâmicas. Partindo-se então, da factibilidade da separação dos efeitos dos domínios físicos (Nashif et al, 1985) e levando-se em conta o Princípio da Superposição FrequênciaTemperatura (PSFT), a função módulo complexo do material viscoelástico pode ser expressa como (Lima et al, 2013):

$$
G\left(\omega_{r}, T_{0}, \delta\right)=F(\delta) G\left(\omega_{r}, T_{0}\right)=G^{\prime}\left(\omega_{r}, T_{0}, \delta\right)+i G^{\prime \prime}\left(\omega_{r}, T_{0}, \delta\right)
$$

onde $\omega_{r}=\alpha_{T}(T) \omega$ designa a frequência reduzida, $\alpha_{T}(T)$ indica fator de deslocamento em temperatura, $\omega$ é a frequência de excitação, $T$ designa a temperatura, $T_{0}$ é a temperatura de referência e $\delta$ denota a deformação estática. $G^{\prime}\left(\omega_{r}, T_{0}, \delta\right), G^{\prime \prime}\left(\omega_{r}, T_{0}, \delta\right)$ e $\eta\left(\omega_{r}, T_{0}, \delta\right)=G^{\prime \prime}\left(\omega_{r}, T_{0}, \delta\right) / G^{\prime}\left(\omega_{r}, T_{0}, \delta\right)$ são, respectivamente, o módulo de armazenamento, o módulo de perda e o fator de perda caracterizados em termos da descrição das propriedades do material sobre condições de sobreposição de cargas estáticas e dinâmicas.

A proposição de uma função $F(\delta)$, contemplativa em linhas gerais das relações entre as deformações estáticas e dinâmicas, inclusiva das relações tensão-deformação específicas à cada dispositivo, incorre à grande número de possibilidades de configurações constitutivas. Alternativamente, para um material elastomérico sujeito à cargas cíclicas lineares sobrepostas a pré-cargas estáticas, para condição de extensão simples em deformações axiais, a seguinte equação de Mooney-Rivlin (Mooney, 1940; Rivlin, 1947) será empregada para a descrição das propriedades viscoelásticas estáticas para os módulos estático e dinâmico combinados:

$$
F(\delta)=C_{1} F_{1}(\delta)+C_{2} F_{2}(\delta)
$$

onde $F_{1}(\delta)=2\left(2 \delta^{2}+1 / \delta\right)$ e $F_{2}(\delta)=2\left(\delta+2 / \delta^{2}\right)$, sendo válida a relação entre as deformações estática e dinâmica: $\delta=\varepsilon+1$. Substituindo (2) na Eq. (1), obtém-se a seguinte aproximação o módulo complexo:

$$
G\left(\omega_{r}, T_{0}, \delta\right)=C_{1} F_{1}(\delta) G\left(\omega_{r}, T_{0}\right)+C_{2} F_{2}(\delta) G\left(\omega_{r}, T_{0}\right)
$$

onde as constantes físicas $C_{1}$ e $C_{2}$ são determinadas a partir da geração de um conjunto de propriedades medidas estaticamente, e $G\left(\omega_{r}, T_{0}\right)$ por um conjunto de propriedades do material comensuradas dinamicamente na ausência de pré-cargas estáticas.

No contexto do estudo numérico que insere, os efeitos induzidos pela pré-carga estática sobre o material viscoelástico são considerados diretamente pela realização de uma análise harmônica estrutural inclusos os efeitos de pré-carregamento estático e as propriedades dinâmicas, são fornecidas pelas seguintes expressões analíticas propostas por Drake e Soovere (1984), para o módulo complexo em frequência reduzida e o fator de deslocamento para o material 
viscoelástico $3 M I S D 112^{T M}$, nos intervalos $210 \leq T \leq 360 \mathrm{~K}$ e $1.0 \leq \omega \leq 1.0 \times 10^{6} \mathrm{~Hz}$, com a temperatura de referência $T_{0}=290 \mathrm{~K}$ :

$$
\begin{gathered}
G\left(\omega_{r}, T_{0}\right)=B_{1}+B_{2} /\left(1+B_{5}\left(i \omega_{r} / B_{3}\right)^{-B_{6}}+\left(i \omega_{r} / B_{3}\right)^{-B_{4}}\right) \\
\log _{10}\left(\alpha_{T}\right)=a\left(\frac{1}{T}-\frac{1}{T_{0}}\right)+2.303\left(\frac{2 a}{T_{0}}-b\right) \log _{10}\left(\frac{T}{T_{0}}\right)+\left(\frac{b}{T_{0}}-\frac{a}{T_{0}^{2}}-S_{A Z}\right)\left(T-T_{0}\right) .
\end{gathered}
$$

Os parâmetros intervenientes às expressões (4) e (5) são listados na Tab. 1.

Tabela 1. Constantes associadas ao módulo complexo e ao fator de deslocamento, respectivamente, do material $3 M I S D 112^{T M}$.

\begin{tabular}{cccccc}
\hline \multicolumn{7}{c}{$B_{1}[\mathrm{MPa}]$} & $B_{2}[\mathrm{MPa}]$ & $B_{3}$ & $B_{4}$ & $B_{5}$ & $B_{6}$ \\
0,4307 & 1200 & 1543000 & 0,6847 & 3,241 & 0,18 \\
\hline \multicolumn{7}{c}{$T^{2}[\mathrm{~K}]$} & $T_{L}[\mathrm{~K}]$ & $T_{H}[\mathrm{~K}]$ & $S_{A Z}[\mathrm{~K}]^{-1}$ & $S_{A L}[\mathrm{~K}]^{-1}$ & $S_{A H}[\mathrm{~K}]^{-1}$ \\
290 & 210 & 360 & 0,05956 & 0,1474 & 0,009725 \\
& $a=\left(D_{B} C_{C}-C_{B} D_{C}\right) / D_{E}$ & $b=\left(D_{C} C_{A}-C_{C} D_{A}\right) / D_{E}$ \\
$C_{A}=\left(1 / T_{L}-1 / T_{0}\right)^{2}$ & \multicolumn{2}{c}{$C_{B}=\left(1 / T_{L}-1 / T_{0}\right)$} & $C_{C}=\left(S_{A L}-S_{A Z}\right)$ \\
$D_{A}=\left(1 / T_{H}-1 / T_{0}\right)^{2} D_{B}=\left(1 / T_{H}-1 / T_{0}\right)$ & $D_{C}=\left(S_{A H}-S_{A Z}\right)$ & $D_{E}=\left(D_{B} C_{A}-D_{A} C_{B}\right)$ \\
\hline
\end{tabular}

\section{AUTOAQUECIMENTO DE MATERIAIS VISCOELÁSTICOS SUJEITOS A CARGAS CICLÍCAS E PRÉ-CARGAS ESTÁTICAS}

De acordo com Rittel (1999), a equação transiente de transferência de calor pode ser escrita como:

$$
q_{g}+k \nabla^{2} T=\rho c_{p} T+(3 \lambda+2 \mu) \alpha T_{0} \varepsilon_{k k}
$$

onde $k, \rho, c_{P}$ e $\alpha$ designam, respectivamente, a condutividade térmica, a densidade, o calor específico por unidade de massa e o coeficiente de dilatação à pressão constante. $\lambda$ e $\mu$ são os coeficientes de Lamé e $\dot{\varepsilon}_{k k}$ o traço da matriz das velocidades de deformação. $\mathrm{O}$ termo $k \nabla^{2} T$ representa a condução de calor, obtido aplicando-se a lei de Fourier (Lienhard e Lienhard, 2004) a um volume infinitesimal do material, e $\rho c_{p} \dot{T}+(3 \lambda+2 \mu) \alpha T_{0} \dot{\varepsilon}_{k k}$ designa o armazenamento de calor e a contribuição termoelástica, resultantes da aplicação da Segunda Lei da Termodinâmica (Gaskell, 2003) e da descrição do campo de pressão em termos da matriz de tensões. A taxa de geração de calor, $q_{g}$, é conotada ao trabalho mecânico dissipado por unidade de tempo $\dot{w}_{m}$, de acordo com a relação constitutiva:

$$
q_{g}=\beta \dot{w}_{m}
$$

onde o coeficiente de rendimento térmico $\beta$ representa a fração do calor gerado pelo efeito da dissipação viscoelástica, dependente das amplitudes e da velocidade das deformações. A parte complementar $(1-\beta) \dot{w}_{m}$ é armazenada no material através de trocas microestruturais (Rittel, 1999).

A potência mecânica dissipada pode ser expressa pelo produto escalar das componentes dos vetores das tensões e das taxas de deformações:

$$
\dot{w}_{m}(t, \omega, T, \delta)=\sigma(t) \dot{\varepsilon}(t, \omega, T, \delta)=G(t, \omega, T, \delta) \varepsilon^{T}(t, \omega, T, \delta)[\bar{C}] \dot{\varepsilon}(t, \omega, T, \delta)
$$


onde $[C](\omega, T, \delta)=G(\omega, T, \delta)[\bar{C}]$ é a matriz de propriedades para um material isotrópico, com dependência a frequência de excitação, a temperatura e a pré-carga estática. Escrevendo-se $\dot{w}_{m}$ para o caso de um estado de tensãodeformação cíclica $\dot{\varepsilon}(t, \omega, T, \delta)=i \omega \mathcal{E}(t, \omega, T, \delta)$, mostra-se que as partes real e imaginária são, respectivamente, proporcionais ao módulo de armazenamento e de perda:

$$
\begin{array}{r}
\dot{w}_{m}(t, \omega, T, \delta)=i \omega \varepsilon^{T}(t, \omega, T, \delta)[\bar{C}] \varepsilon(t, \omega, T, \delta) G^{\prime}(\omega, T, \delta)- \\
\omega \varepsilon^{T}(t, \omega, T, \delta)^{T}[\bar{C}] \varepsilon(t, \omega, T, \delta) G^{\prime \prime}(\omega, T, \delta) .
\end{array}
$$

Substituindo $\varepsilon(t, \omega, T, \delta)$ por uma variação senoidal da forma $\varepsilon_{0}(t, \omega, T, \delta) \sin (\omega t+\varphi)$, sobreposta à uma précarga estática $\delta$, é possível representar a contribuição da parte imaginária do módulo complexo à dissipação viscoelástica de energia, enquanto a parte associada ao armazenamento puramente elástico anula-se sobre um ciclo de vibração:

$$
\dot{w}_{m}(t, \omega, T, \delta)=-\omega G^{\prime \prime}(\omega, T, \delta) \varepsilon_{0}(t, \omega, T, \delta)^{T}[\bar{C}] \varepsilon_{0}(t, \omega, T, \delta) \sin ^{2}(\omega t+\varphi)
$$

onde $\varphi$ é a fase. De acordo com (10), a potência viscoelástica dissipada não é constante variando sobre um ciclo de vibração com a função $\sin ^{2}(\omega t+\varphi)$, sendo esta variação dependente da amplitude das deformações para cada instante de tempo. A utilização direta desta equação para o cálculo da taxa de geração de calor resulta em um alto custo computacional, sendo necessário, aproximadamente 10 passos de tempo por período para a integração temporal (Cazenove et al., 2010).

Segundo Merlette (2005), a diferença entre as velocidades dos fenômenos estruturais e térmicos é suficientemente grande para que um período correspondente a um ciclo de vibração leve à fenômenos de troca e armazenamento de calor que resultem a um aumento significativo. Neste sentido, é passível a substituição do termo $\sin ^{2}(\omega t+\varphi)$ pelo valor médio da função seno quadrático, conduzindo a:

$$
\dot{w}_{m}(t, \omega, T, \delta)=-\frac{1}{2} \omega G^{\prime \prime}(\omega, T, \delta) \varepsilon_{0}(t, \omega, T, \delta)^{T}[\bar{C}] \varepsilon_{0}(t, \omega, T, \delta)
$$

Do ponto de vista do balanço de energia térmica, o calor gerado pelo efeito viscoelástico de dissipação corresponde por uma quantidade de energia recebida pelo material e, portanto, deve ser expressa positiva. Logo, a inclusão da Eq. (11) em (7), resulta na seguinte expressão para a fonte de calor:

$$
q_{g}(\omega, T, \delta)=\left|\beta \dot{w}_{m}(\omega, T, \delta)\right|=\frac{1}{2} \beta \omega G^{\prime \prime}(\omega, T, \delta) \varepsilon_{0}^{T}(\omega, T, \delta)[\bar{C}] \varepsilon_{0}(\omega, T, \delta) .
$$

Introduzindo a relação (12) em (6), expressa-se o autoaquecimento interno de materiais viscoelásticos para a condição de sobreposição de cargas dinâmicas à cargas estáticas, desprezada a contribuição termoelástica para estados de tensão-deformação cisalhantes:

$$
\frac{1}{2} \beta \omega G^{\prime \prime}(\omega, T, \delta) \varepsilon_{0}^{T}(\omega, T, \delta)[\bar{C}] \varepsilon_{0}(\omega, T, \delta)+k \nabla^{2} T=\rho c_{p} \dot{T}
$$

O efeito da temperatura sobre o comportamento dinâmico viscoelástico é devido unicamente à dependência das propriedades do material, pressupondo-se que a resposta estrutural transiente é rapidamente atenuada. Assim, o calor gerado pela dissipação viscoelástica pode ser expresso apenas como um carregamento térmico adicional na análise térmica estrutural (Cazenove et al, 2012), sendo calculado para cada elemento viscoelástico integrando-se a Eq. (12) sobre o volume de cada elemento viscoelástico:

$$
q_{g}(\omega, T, \delta)=\frac{\beta \omega \eta(\omega, T, \delta)}{2 V_{(e)}} U_{(e)}^{T}(\omega, T, \delta)\left[K_{v}^{\prime}(\omega, T, \delta)\right] U_{(e)}(\omega, T, \delta)
$$

onde $\left[K_{v}(\omega, T, \delta)\right]=G^{\prime}(\omega, T, \delta) \int_{V(e)}[B]^{T}[\bar{C}][B] d V$ é a parte real da matriz de rigidez viscoelástica, $[B]$ é a matriz de 
deformação-deslocamento, $V_{(e)}$ o volume elementar e $U_{(e)}(\omega, T, \delta)$ representa o vetor do campo de deslocamentos a nível elementar.

\section{SIMULAÇÕES NUMÉRICAS}

\subsection{Incorporação do comportamento viscoelástico no modelo de elementos finitos e resolução iterativa sequencial}

Para a incorporação do comportamento viscoelástico em elementos finitos, assume-se que a matriz de rigidez complexa da estrutura pode ser decomposta em (Lima et al., 2013):

$$
\left[K^{*}(\omega, T, \delta)\right]=\left[K_{e}\right]+\left[K_{v}^{*}(\omega, T, \delta)\right]
$$

onde $\left[K_{e}\right]$ representa a matriz de rigidez associada a parte puramente elástica e $\left[K_{v}^{*}(\omega, T, \delta)\right]$ é a matriz de rigidez viscoelástica, associada aos elementos viscoelásticos. Assumindo que o coeficiente de Poisson independe da frequência de excitação e da temperatura, o módulo complexo pode ser fatorado da matriz de rigidez viscoelástica (Lima et al., 2005):

$$
\left[K_{v}^{*}(\omega, T, \delta)\right]=G(\omega, T, \delta)\left[\bar{K}_{v}\right]=G^{\prime}(\omega, T, \delta)(1+i \eta(\omega, T, \delta))\left[\bar{K}_{v}\right]
$$

onde $\left[\bar{K}_{v}\right]$ é a matriz de rigidez fatorada da subestrutura viscoelástica, com a parte imaginária $\left[K_{v}^{*}(\omega, T, \delta)\right]$ proporcional ao amortecimento do sistema.

Para a implementação computacional da capacidade dissipativa do material viscoelástico, define-se uma matriz de amortecimento viscoso equivalente proporcional à $\left[\bar{K}_{v}\right]$, formulada como (Lima et al., 2005):

$$
\left[C_{e q}(\omega, T, \delta)\right]=\frac{\eta(\omega, T, \delta) G^{\prime}(\omega, T, \delta)}{\omega}\left[\bar{K}_{v}\right]=\alpha(\omega, T, \delta)\left[\bar{K}_{v}\right]
$$

Desta forma, a equação do movimento no domínio da frequência para um amortecedor viscoelástico contendo $N$ graus de liberdade, pode ser escrita como:

$$
\left(\left[K_{e}\right]+G^{\prime}(\omega, T, \delta)\left[\bar{K}_{v}\right]+i G^{\prime \prime}(\omega, T, \delta)\left[\bar{K}_{v}\right]-\omega^{2}[M]\right)\{U(\omega, T, \delta)\}=F(\omega) .
$$

Como o acoplamento direto entre o problema mecânico e térmico, resulta em um alto custo computacional em termos de convergência (Gopalakrishna e Lai, 1998), leva-se o problema ao acoplamento termomecânico fraco ou sequencial (Merlette, 2005), descrevendo-se o problema termoviscoelástico acoplado pelo seguinte sistema matricial:

$$
\left[\begin{array}{ll}
{[0]} & {[0]} \\
{[0]} & {[A]}
\end{array}\right]\left\{\begin{array}{c}
\{\dot{U}(\omega, T, \delta)\} \\
\{\dot{T}\}
\end{array}\right\}+\left[\begin{array}{cc}
{\left[K^{*}(\omega, T, \delta)\right]-\omega^{2}[M]} & {[0]} \\
{[0]} & {[W]}
\end{array}\right]\left\{\begin{array}{c}
\{U(\omega, T, \delta)\} \\
\{T\}
\end{array}\right\}=\left\{\begin{array}{c}
\{F(\omega)\} \\
\left\{q_{C}\right\}+\left\{q_{\mathrm{g}}\right.
\end{array}\right\}
$$

onde $[A]$ e $[W]$ são, respectivamente, as matrizes de amortecimento e rigidez térmica, formuladas com base às funções de forma para elementos térmicos, no calor específico e na condutividade térmica do material. $\left\{q_{C}\right\}$ e $\left\{q_{\mathrm{g}}\right\}$ representam os vetores de carregamentos térmicos decorrentes da convecção externa e da dissipação viscoelástica.

Como $\left\{q_{\mathrm{g}}\right\}$ é obtido a partir da energia de deformação resultante da resposta estrutural, e as matrizes de rigidez viscoelástica e de amortecimento equivalente dependem do campo de temperatura, é necessária à utilização de um procedimento iterativo de resolução, no qual, para um dado incremento de tempo $\Delta t$, a análise harmônica estrutural com inclusão dos efeitos de pré-cargas estática é realizada, avaliando-se em sequência as fontes de calor para os elementos correspondentes, obtendo em seguida, o campo de temperatura pela realização da análise térmica transiente, para a então atualização das matrizes estruturais em $t+\Delta t$, até que seja atingido o tempo final de aplicação do carregamento cíclico. Assim sendo, a metodologia de simulação do fenômeno do autoaquecimento, foi implementada seguindo esta sequência de realizações na linguagem APDL (Ansys Parametric Design Language), integrada ao software de elementos finitos $A N S Y S^{\mathrm{TM}}$, com interface ao ambiente de programação $M A T L A B^{\circledR}$. 


\subsection{Resultados Numéricos}

Para a investigação do fenômeno da fuga térmica e a avaliação da influência dos efeitos da pré-carga estática sobre o fenômeno do autoaquecimento interno de materiais viscoelásticos, as simulações numéricas foram realizadas para o dispositivo translacional viscoelástico apresentado por Lima et al (2013), composto por duas camadas viscoelásticas do material 3 M ISD $112^{\mathrm{TM}}$ entre três placas de aço SAE 1020 mostrado na Fig. 1. Considerado o plano de simetria indicado, a junta foi modelada utilizando os seguintes elementos:

- Elemento tridimensional SOLID45, com oito nós e três graus de liberdade por nó - deslocamentos nas direções $\mathrm{x}, \mathrm{y}$ e $\mathrm{z}$;

- Elemento compatível SOLID70, com o mesmo número de nós e um grau de liberdade por nó - temperatura. As propriedades térmicas utilizadas nas simulações termomecânicas são expostos na Tab. 2

$\mathrm{O}$ dispositivo foi considerado termicamente isolado na face indicativa do plano de simetria, admitindo uma transferência de calor por convecção natural, com o coeficiente de convecção natural $h=13,016 \mathrm{~W} /\left(\mathrm{m}^{2} \mathrm{~K}\right)$ (Lima et al., 2013) e a temperatura ambiente $T_{\infty}=25^{\circ} \mathrm{C}$.

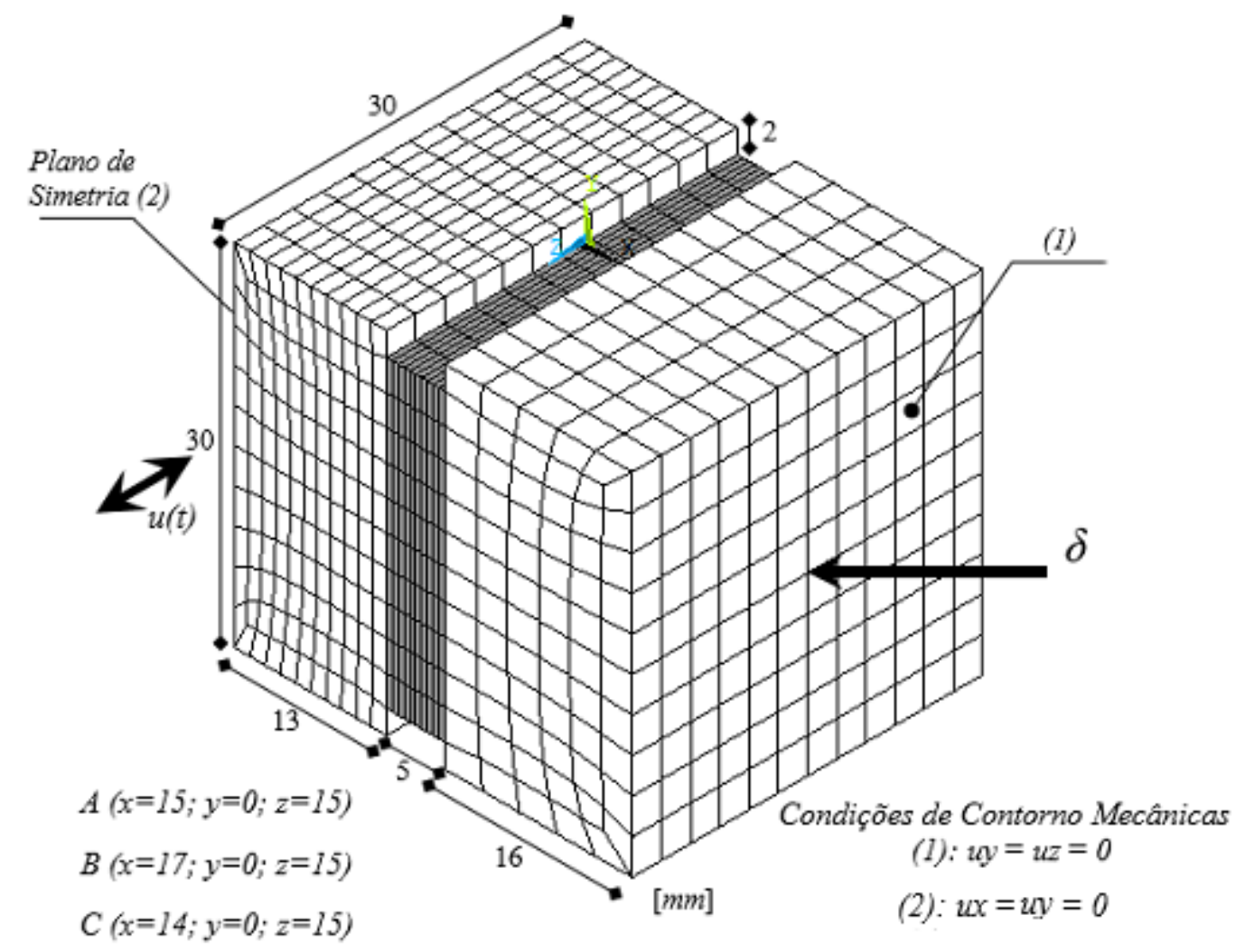

Figura 1. Características geométricas e condições de contorno mecânicas aplicadas.

Tabela 2. Propriedades térmicas utilizadas nas simulações numéricas.

\begin{tabular}{ccccc}
\hline Material & $\rho\left[\mathrm{kg} \cdot \mathrm{m}^{-3}\right]$ & $c_{p}\left[\mathrm{~J} \cdot \mathrm{kg}^{-1} \cdot \mathrm{K}^{-1}\right]$ & $k\left[\mathrm{~W} \cdot \mathrm{m}^{-1} \cdot \mathrm{K}^{-1}\right]$ & $\beta$ \\
\hline$I S D 112$ & 1100 & 2000 & 0,16 & 0,1755 \\
\hline$S A E 1020$ & 7850 & 476 & 35 & 0 \\
\hline
\end{tabular}

A análise termoviscoelástica em termos da determinação da evolução da temperatura nos pontos $A, B$ e $C$ indicados na Fig.1 , abrange a fase de aplicação do carregamento dinâmico ao longo de $3396 s$, caracterizado pelo deslocamento cíclico $u(t)=u_{0} \sin \left(2 \pi f_{0} t\right)$, com $u_{0}=3,5 \mathrm{~mm}$ e $f_{0}=14 \mathrm{~Hz}$ para três diferentes valores de pré-carga estática, distinguindo-se os quatro respectivos cenários de teste para o fenômeno da fuga térmica: $(a) \delta=0 \mathrm{~N},(b) \delta=100 \mathrm{~N}$, (c) $\delta=200 \mathrm{~N}$ e (d) $\delta=250 \mathrm{~N}$. Na Figura 2(a) é exposta a distribuição de temperatura para o cenário $(a)$ nos pontos $A, B$ e $C$, e a Fig. 2(b) viabiliza a comparação da evolução da temperatura interna do material no ponto $A$, para os quatro cenários de teste.

O comportamento evidenciado para a fase de carregamento, configura o fenômeno da fuga térmica, uma vez que a temperatura do material sofre aumentos significativamente grandes em instantes de tempo que caracteristicamente 
indicam a convergência a uma temperatura estacionária para a configuração de equilíbrio térmico. O aumento abrupto e o singular comportamento da evolução da temperatura para os primeiros instantes de ciclagem do dispositivo e a tendência ao alcance de uma temperatura de equilíbrio superior, para os instantes que antecedem a retirada do carregamento, remete à descrição feita por Lesieutre e Govindswamy (1996).
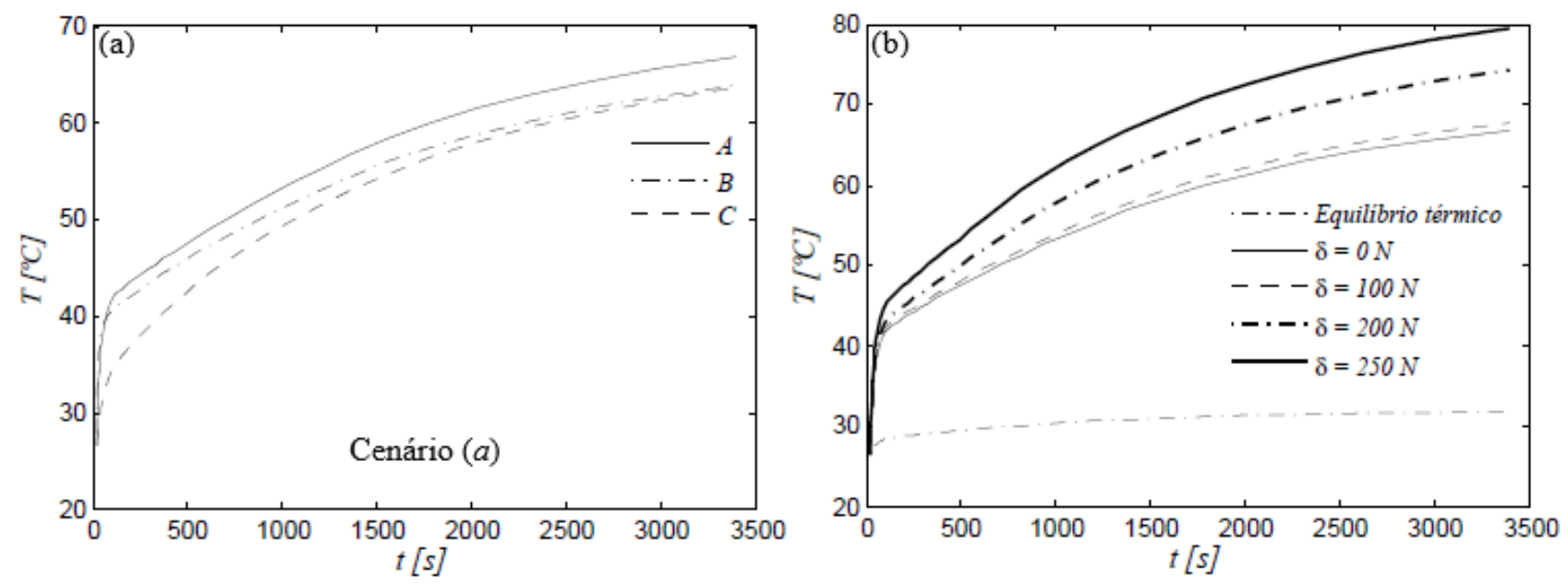

Figura 2. (a) Evolução da temperatura nos pontos $A, B$ e $C$ para o cenário de teste $(a)$; (b) Evolução da temperatura no ponto $A$ para os cenários $(a),(b),(c)$ e $(d)$.

O aumento constatado entre os cenários de teste, Fig. 2(b), resultam unicamente da inclusão da pré-carga estática, sendo que os valores obtidos para o campo de temperatura, comprometem em totalidade as propriedades dissipativas, com drástica perda da capacidade de amortecimento e significativo aumento da rigidez, para os cenários $(b),(c)$ e $(d)$, recorrendo no aumento da amplitude das deformações e do aquecimento do material. Em razão da não homogeneidade das condições de transferência de calor, verifica-se para pontos distintos do material, diferentes valores para o campo de temperatura do material, Fig. 2(a), consistentemente como já relatado na literatura.

\section{ESTUDO EXPERIMENTAL}

Esta seção é dedicada à descrição do procedimento operacional utilizado para a verificação experimental do fenômeno da fuga térmica em uma junta translacional viscoelástica. A Figura 3 mostra a montagem experimental completa, com a junta viscoelástica construída de acordo com as dimensões geométricas apresentadas na Fig. 1, composta por duas camadas de 5 milímetros do material $3 M V H B 4910^{\mathrm{TM}}$ inseridas entre três blocos de aço conectados a uma estrutura rígida, a qual é acoplada à uma máquina universal de ensaios $M T S 800^{\mathrm{TM}}$. A temperatura da camada viscoelástica nos pontos $A, B$ e $C$, com as posições ora pré-definidas, foram medidas por meio de termopares. Os sinais de tensão foram adquiridos e processados utilizando um analisador de sinais Agilent ${ }^{\mathrm{TM}} 34970$. O carregamento dinâmico do dispositivo, foi conseguido aplicando-se um deslocamento cíclico vertical, $u(t)=u_{0} \sin \left(2 \pi f_{0} t\right)$, com $u_{0}=3,5 \mathrm{~mm}$ e $f_{0}=14 \mathrm{~Hz}$, durante $3396 \mathrm{~s}$, em sobreposição à deslocamentos estáticos aplicados ao corpo de prova por intermédio de parafusos, tal como elucidado na Fig. 3.

Para a aplicação do carregamento estático foi utilizada uma estratégia para à estimação dos valores dos deslocamentos estáticos a serem aplicados na base da estrutura rígida, baseada no conceito de matriz de rigidez tangente para a representação do comportamento estático do material viscoelástico, e na pressuposição de que apenas as partes viscoelásticas são deformados durante a aplicação da pré-carga estática (Lima et al., 2013). Assim, tendo em conta a área da seção transversal da camada viscoelástica, $A_{v}=26 \times 30 \mathrm{~mm}^{2}$, e a relação para os módulos longitudinal e transversal puramente elásticos, $E_{0}=2 G_{0}(1+v)$, os deslocamentos estáticos correspondentes foram estimados pela relação, $\Delta=F_{0} t_{v} /\left(2 A_{v} G_{0}(1+v)\right)$, onde $v=0,49$ é o coeficiente Poisson, $t_{v}=0,005 \mathrm{~mm}$ a espessura da camada viscoelástica e $F_{0}$ o valor da pré-carga estática utilizada na simulação numérica, configurando, portanto, os seguintes cenários de investigação: (a) $\Delta=0,0 \mathrm{~mm}$ e (b) $\Delta=1,0 \mathrm{~mm}$.

Os valores dos parâmetros de convecção natural $h$ e de conversão térmica $\beta$ utilizados nas simulações numéricas foram assumidos de acordo com Lima et al. (2013). No entanto, estes variam em um determinado intervalo em função de várias condições, tais como a geometria da composição viscoelástica e, das amplitudes das deformações estáticas e dinâmicas, esperando-se que os valores admitidos não se adequassem às condições específicas ensaiadas, o que inviabiliza a comparação direta dos resultados numéricos e experimentais. Neste contexto, intentou-se a consecução de um procedimento de identificação paramétrica para a determinação simultânea dos valores de $h$ e $\beta$ para as condições 
de ensaio consideradas, procedendo de forma, que obtido o valor ótimo do coeficiente de convecção natural para o primeiro cenário de teste, este é assumido como constante para o cenário posterior, admitidas as mesmas condições ambientais de realização dos testes.

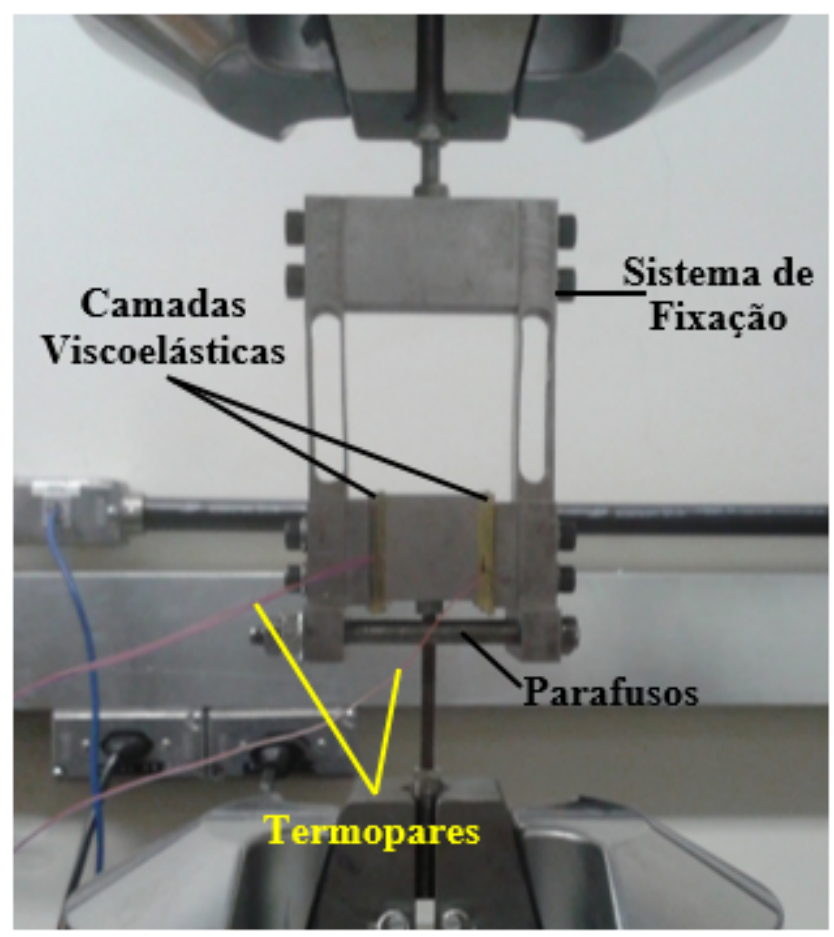

Figura 3. Junta viscoelástica translacional montada na máquina universal de ensaios $M T S 800^{T M}$.

Neste sentido, a estratégia de otimização empregada, consiste da minimização da função objetivo, $F_{o b j}=\sum_{j=1}^{n_{(\exp )}}\left(T_{A}^{(\exp )}\left(t_{j}\right)-T_{A}^{(s)}\left(t_{j}\right)\right)^{2}$, onde $T_{A}^{(\exp )}\left(t_{j}\right)$ e $T_{A}^{(s)}\left(t_{j}\right)$ representam, respectivamente, as temperaturas experimentais e numéricas no ponto $A$, no instante de tempo $t_{j}$, com $n_{(\exp )}$ designando o número de pontos considerados nos resultados experimentais, pela técnica Colônia de Vagalumes, bioinspirada na característica de bioluminescência destes insetos coleópteros auto organizáveis, luminosamente auto emissivos e descrita por equações que modelam a atratividade, a luminosidade e a movimentação em relação à distância entre indivíduos. O problema de otimização como definido consta com apenas restrições laterais, delineadas pelos limites das variáveis de projeto, caracterizadas pelos seguintes intervalos relativos a cada cenário de teste: (a) $h:[13-50] \mathrm{W} / \mathrm{m}^{2} \mathrm{~K}$, $\beta:[0,1-0,9]$; e (b) $\beta:[0,1-0,9]$.

Os parâmetros utilizado para o Colônia de Vagalumes são os que constam: coeficiente de absorção de luz pelo meio $\gamma=1$, fator de atratividade $\varphi_{0}=0,2$ e o parâmetro de inserção de aleatoriedade no caminho percorrido pelo vagalume $\alpha=0,5$. Foi utilizada uma população de vagalumes com 100 indivíduos, sendo o número de gerações limitado a 50 iterações, resultando em 5000 avaliações da função objetivo. Para a adequação da quantidade de pontos resultantes da simulação numérica ao número de pontos experimentais, destaca-se a utilização da interpolação Polinomial de Hermite (Yang et al., 2005).

Na Figura 4 são expostas as evoluções de temperatura preditas pelo modelo ajustado com os valores identificados em contrapartida aos resultados experimentais. Os resultados remetem qualitativamente à evolução da temperatura obtida nas simulações numéricas, confirmando a influência da pré-carga estática no fenômeno da fuga térmica. Constata-se, que para ambos os cenários de teste, as correlações entre as previsões numéricas e as temperaturas medidas experimentalmente são muito satisfatórias. Além disso, evidencia-se que com a inserção da pré-carga estática, a taxa de conversão térmica é reduzida. Isto se deve ao fato que uma quantidade mais significativa da parte complementar da energia mecânica dissipada é armazenada no material, em consonância com o pronunciamento das modificações de sua microestrutura devido à inserção da deformação estática. Ainda, os valores ótimos obtidos para o parâmetro de conversão térmica, permaneceram no intervalo $[0,1-1,0]$, qualitativamente consistente com o relatado por Rittel (2000) e Lima et al. (2013). Entretanto, observa-se em ambos os casos, a nítida estabilização a uma temperatura de equilíbrio não evidenciada para as simulações numéricas. Isto é devido à potencialização da evacuação do calor gerado, causada pelo fluxo de ar criado em torno do corpo de prova pelo movimento de ciclagem vertical. Esta consideração é ainda reforçada pelo o alto valor do coeficiente de convecção natural obtido, no ajuste do modelo. 

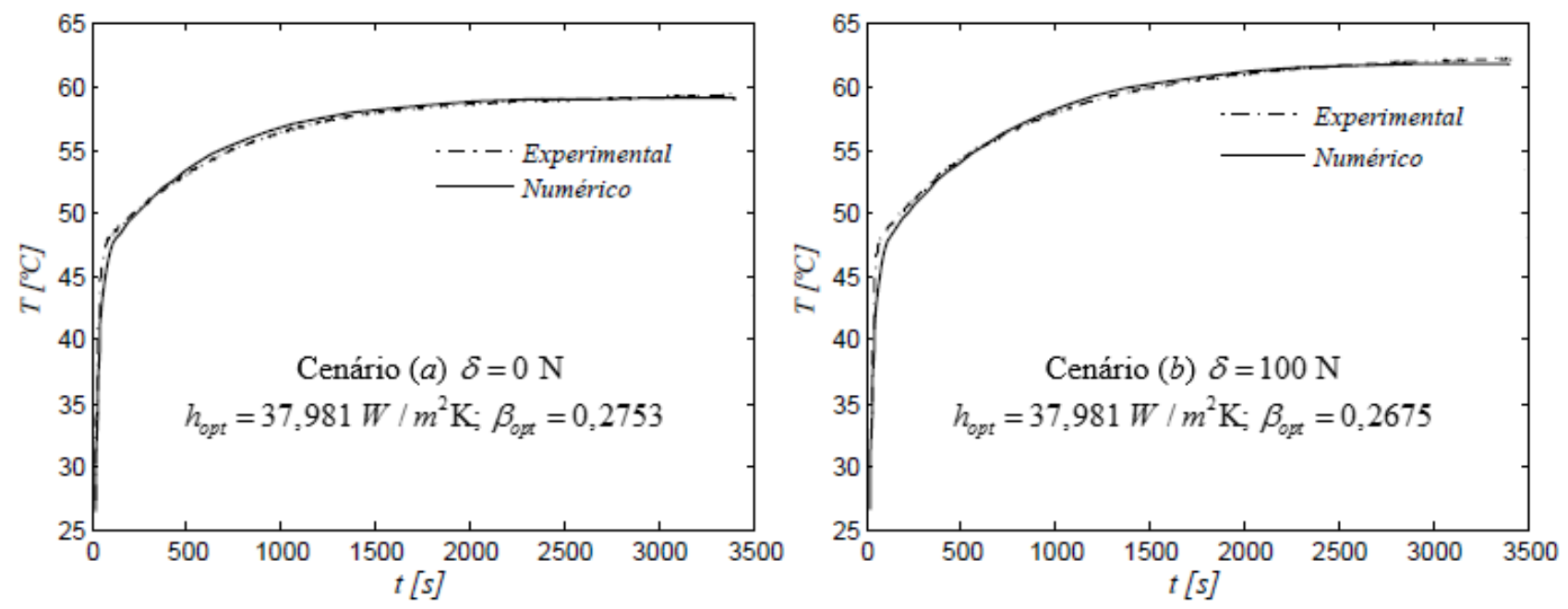

Figura 4. Comparativo entre as temperaturas experimentais e simuladas para os valores ótimos de $h$ e $\beta$ para os cenários $(a)$ e $(b)$.

\section{CONCLUSÕES}

O processo de modelagem e simulação numérica do autoaquecimento interno em materiais viscoelásticos apresentado, consiste da extensão da metodologia proposta por Cazenove et al. (2012) à contemplação dos efeitos induzidos por pré-cargas estáticas em dispositivos viscoelásticos mais complexos de interesse industrial (Lima et al., 2013).

No contexto das simulações numéricas, os resultados viabilizaram a evidência da influência da pré-carga na estática na evolução da temperatura interna do material viscoelástico, destacando-se a estratégia utilizada para incorporação da mesma no comportamento viscoelástico, a partir de um ponto de vista prático de engenharia, com potencialidade de utilização em conjunto com outros softwares comerciais de elementos finitos. Além disso, o comportamento do campo de temperatura obtido para a simulação do fenômeno da fuga térmica se mostrou qualitativamente muito condizente com o relatado por Lesieutre e Govindswamy (1996).

O procedimento de identificação paramétrica implementado com base a técnica Colônia de Vagalumes, integrando a interpolação Polinomial de Hermite, se mostrou muito satisfatório no ajuste do modelo, o que viabilizou a comparação dos resultados da simulação aos obtidos experimentalmente, confirmando a maioria das evidências numéricas. Neste ponto, vale salientar que a influência do fluxo de ar gerado pelo processo de ciclagem vertical sobre a convecção natural, está sendo investigada pelos autores, com vista ao desenvolvimento de uma estratégia para a minimização deste efeito.

\section{AGRADECIMENTOS}

À CAPES pela bolsa de estudos de L. F. F. Rodovalho, ao CNPQ pela bolsa de pesquisa e ao apoio financeiro aos projetos de A. M. G. de Lima, À FAPEMIG pelo apoio financeiro aos projetos de pesquisa, ao Laboratório de Mecânica de Estruturas Prof. José Eduardo Tannús Reis (LMEst) da Faculdade de Engenharia Mecânica da Universidade Federal de Uberlândia e ao Instituto Nacional de Ciência e Tecnologia de Estruturas Inteligentes em Engenharia (INCT-EIE).

\section{REFERÊNCIAS}

Cazenove, J. de; Lima, A. M. G., Rade, D. A., Araújo, C. A., 2010. Computacional modeling and experimental validation of self-heating effects in viscoelastic materials. VI NATIONAL CONGRESS OF MECHANICAL ENGINEERING - CONEM 2010, Campina Grande - PB, Brazil.

Cazenove, J. de, Rade, D. A., Lima, A. M. G., Araújo, C. A., 2012. A numerical and experimental investigation on selfheating effects in viscoelastic dampers. Mechanical Systems and Signal Processing, 27, pp. 433-445.

Drake, M. L., Soovere, J., 1984. A design guide for damping of aerospace structures, in: AFWAL Vibration Damping Workshop Proceedings 3, Atlantic City USA.

Gaskell, D. R., 2003. Introduction to the Thermodynamics of Materials - Fourth Edition. Taylor e Francis, 640 p., ISBN 978-1591690139.

Gopalakrishna, H. S., Lai, M. L., 1998. Finite elemento heat transfer analysis of viscoelastic damper for wind applications. Journal os Wind Engineering and Industrial Aerodynamics, 77\&78, pp. 283-295.

Lesieutre, G. A., and Govindswamy, K. M., 1996. Finite Element Modeling of Frequency-Dependent and TemperatureDependent Dynamic Behavior of Viscoelastic Materials in Simple Shear. International Journal of Solids and Structures, Vol. 33, No. 3, pp. 419-432. 
Lienhard, J. H. IV, Lienhard, J. H. V, 2004. A heat transfer textbook - Third edition, Phlogiston Press, Cambridge MA, E.U.A.

Lima, A. M. G., Rade, D. A., 2005. Modeling of structures supported on viscoelastic mounts using FRF substruturing. Twelfth International Congress on Sound and Vibration, Lisbon, Portugal.

Lima, A. M. G., Rade, D. A., Lacerda, H. B., Araújo, C. A., 2013. Influence of the Combined Dynamic and Statics Strains on the Self-Heating Phenomenon in Viscoelastic Dampers. 22nd International Congress of Mechanical Engineering - COBEM 2013, Ribeirão Preto-SP, Brazil, 9 p.

Merlette, N., 2005. Amortissement des caisses automobiles par des films minces viscoélastiques pour l'amélioration du confort vibratoire. Thése de doctorat - Ecole Centrale de Lyon, Lyon, France, 219 p.

Mooney, M., 1940. A theory of large elastic deformations. Journal of Applied Physics 11, pp. 582-592.

Nashif, A. D., Jones, D. I. G., Henderson, J. P., 1985. Vibration Damping. John Wiley \& Sons, New York, E.U.A, ISBN 978-0471867722.

Rittel, D., 1999. On the conversion of plastic work to heat during high strain rate deformation of glassy polymers. Mechanics of Materials 31, pp. 131-139.

Rittel, D., 2000. An investigation of the heat generated during cyclic loading of two glassy polymers. Part II: Thermal Analysis. Journal of Mechanics of Materials 32, pp. 149-159.

Rivlin, R. S., 1947. Torsion of a rubber cylinder. Journal of Applied Physics 18, pp. 444-449.

Yang, W. Y., Cao, W., Chung, T. S., Morris, J., 2005. Applied numerical methods using MATLAB ${ }^{\circledR}$. John Wiley \& Sons, New Jersey, E.U.A., ISBN 0-471-69833-4.

Yang, X.-S., 2008. Firefly Algorithm. Nature-Inspired Metaheuristic Algorithms. Luniver Press: Cambridge.

\title{
RESPONSABILIDADE AUTORAL
}

"Os autores são os únicos responsáveis pelo conteúdo deste trabalho".

\section{A NUMERICAL-EXPERIMENTAL INVESTIGATION OF THE INFLUENCE OF STATIC PRELOAD TO COMBINED THE CYCLIC LOADS IN SELF-HEATING IN VISCOELASTIC MATERIALS}

\author{
Luiz Fernando Ferreira Rodovalho, luiz.ffrodovalho@gmail.com ${ }^{1}$ \\ Antonio Marcos Gonçalves de Lima, amglima@ mecanica.ufu.br ${ }^{1}$ \\ Romes Antonio Borges, romes.borges@catalao.ufg.br ${ }^{2}$ \\ Núbia dos Santos Saad, nubia@ufu.br ${ }^{1}$ \\ Helder Barbieri Lacerda, helder@mecanica.ufu.br ${ }^{1}$
}

\begin{abstract}
${ }^{1}$ Federal University of Uberlandia - School of Mechanical Engineering, Campus Santa Mônica, Ave. João Naves de Ávila, 2121, P. O. Box. 593, CEP. 38400-902, Uberlândia-MG, Brazil.

${ }^{2}$ Federal University of Goiás - Departament of Mathematics and Industrial Mathematics, Regional Catalão, Ave. Dr. Lamartine Pinto de Avelar, 1120, CEP. 75704-020, Catalão-GO, Brazil.
\end{abstract}

\begin{abstract}
Due to the numerous possible applications in engineering, the search for broad technological dominance over the internal damping mechanism of viscoelastic materials, refers to the development of methodologies for modeling and numerical simulation tools, more effective and inclusive of all effects induced by operational and environmental parameters.In the sense, it is the initial design or pre-project stage of the systems incorporating viscoelastic components, the self-heating phenomenon, often neglected in most traditional analysis procedures, should be taken into account in the characterization and prediction of structural behavior, therefore, can represent significant losses in performance and significant changes in the dynamic response of the structure in question. For applications involving the simultaneous submission of the viscoelastic material a static preload combined with cyclic dynamic loads, such as cushions motors and configurations of isolation or damping related to high frequencies, such procedures can lead to poor designs or even the serious flaws, since the energy dissipation of the material leads to elevation of the internal temperature of the same. In this work, effective a numerical and experimental investigation of the effects of static preload on the self-heating phenomenon in viscoelastic dampers cyclically loaded, specifically aiming at thermal runaway phenomenon for a full triaxial stress state. After the presentation of the main theoretical aspects of the methodology, numerical and experimental results in terms of the temperature will be compared for different levels of static preloads.
\end{abstract}

Keywords: Viscoelastic materials, self-heating, thermoviscoelasticity, thermal runaway. 